\title{
Risk Factors for Severe Hyponatremia Related to Cisplatin: A Retrospective Case-Control Study
}

\author{
Shiro Hatakeyama, ${ }^{*, a, b}$ Toshihiro Shida, ${ }^{a}$ and Hiroaki Yamaguchi ${ }^{a, b}$ \\ ${ }^{a}$ Division of Pharmacy, Yamagata University Hospital; 2-2-2 Iida-nishi, Yamagata 990-9585, Japan: and \\ ${ }^{b}$ Department of Pharmaceutical Science, Faculty of Medicine, Yamagata University; 2-2-2 Iida-nishi, Yamagata \\ 990-9585, Japan.
}

Received June 7, 2019; accepted August 27, 2019

\begin{abstract}
Onset of severe hyponatremia following cisplatin (CDDP) administration has been previously reported. However, the risk factors associated with hyponatremia still remain unclear. We conducted a retrospective, single-center, case-control study to identify independent risk factors of severe hyponatremia in patients with various types of cancers. Adult patients who received intravenous CDDP administration between January 2012 and December 2017 met the inclusion criteria. The investigators recorded patients' demographics and clinical information retrospectively, and assessed the lowest serum sodium level within $21 \mathrm{~d}$ of the first CDDP administration. Risk factors for grade $\geq 3$ hyponatremia were examined via a logistic regression analysis. Among a total of 472 patients, fifty patients (10.6\%) developed grade $\geq 3$ hyponatremia. Univariate analysis revealed that age ( $\geq 65$ years), presence of small cell lung or esophageal cancer, and lower sodium concentrations in the serum $(<138 \mathrm{mEq} / \mathrm{L})$ were significantly associated with grade 3 and 4 hyponatremia. Additionally, multivariable logistic regression analysis showed that the presence of small cell lung cancer (adjusted odds ratio, 3.26; 95\% confidence interval (CI), 1.07-10.00) and lower sodium concentrations in the serum $(<138 \mathrm{mEq} / \mathrm{L})$ (adjusted odds ratio, 6.18; 95\%CI, 3.21-11.90) were independent risk factors of grade 3 and 4 hyponatremia. Thus, serum sodium concentrations in patients with these risk factors should be closely monitored after CDDP administration.
\end{abstract}

Key words hyponatremia; cisplatin; risk factor; adverse event

\section{INTRODUCTION}

Hyponatremia is one of the most common electrolyte disorders in hospitalized patients, and is caused by several factors, such as the use of some anticancer agents. ${ }^{1)}$ Severe hyponatremia causes some serious complications, including convulsion, respiratory distress, neurologic disturbance, and coma. ${ }^{1)}$ Hyponatremia was also frequently observed in cancer patients. Previous studies reported that hyponatremia $(<135 \mathrm{mEq} / \mathrm{L})$ was present in $47 \%$ of newly admitted cancer patients, ${ }^{2)}$ and $3.7-33.8 \%$ of cancer patients had serum sodium concentrations below $130 \mathrm{mEq} / \mathrm{L}^{3,4)}$ In particular, prostate, pancreatic, and small cell lung cancers were frequently associated with the onset of hyponatremia.)

Administration of cisplatin (CDDP), a platinum anticancer agent, has been related to the development of hyponatremia, although renal failure, nausea, vomiting, and ototoxicity were the major adverse events. ${ }^{5-7)}$ Previous studies indicated that some mechanisms, including syndromes of inappropriate secretion of antidiuretic hormone (SIADH) and renal salt wasting (RSW), caused hyponatremia following CDDP administration. ${ }^{8,9)}$

Thus, early detection and treatment of severe hyponatremia following CDDP administration are required to prevent serious complications. A recent study identified some risk factors for hyponatremia following CDDP by univariate analysis and reported that the frequency of severe hyponatremia was different for each type of cancer. ${ }^{10)}$ However, independent risk factors still remain unclear. Therefore, this retrospective, casecontrol study was conducted to elucidate the independent risk factors for severe hyponatremia, following first CDDP administration among patients with different cancer types.

\section{PATIENTS AND METHODS}

Study Design This retrospective, case-control study was conducted at the Yamagata University Hospital. The study protocol complied with the ethical guidelines for epidemiological studies and was approved by the institutional review board of Yamagata University (approval No. 2018-427).

Patients Patients who received intravenous administration of CDDP between January 2012 and December 2017 at Yamagata University Hospital were initially considered for the study. The exclusion criteria included patients $<18$ years of age, who previously received CDDP chemotherapy, who were receiving CDDP chemotherapy with a high dose of methotrexate, who were receiving daily, weekly, or bi-weekly CDDP administration. Among the enrolled patients, those who suffered from hyponatremia with a grade $\leq 1$ were included in the control group; meanwhile, those who developed hyponatremia of grade 3 or 4 were considered the experimental group. The onset of hyponatremia was assessed using the lowest serum sodium level within $21 \mathrm{~d}$ of the first CDDP administration. Hyponatremia was graded based on version 4.0 of the Common Terminology Criteria for Adverse Events (CTC AE v4.0), as grade $1(137-130 \mathrm{mEq} / \mathrm{L})$, grade $3(129-120 \mathrm{mEq} / \mathrm{L})$ and grade $4(<120 \mathrm{mEq} / \mathrm{L})$. There is no grade 2 hyponatremia in the CTCAE v4.0.

Data Collection The investigators recorded the patients' demographics and clinical information, including age, sex, 
tumor type, CDDP dose, number of concomitant anticancer drugs, creatinine clearance, serum albumin levels, serum sodium concentration, frequency of measuring sodium concentrations, hydration regimen on the day of CDDP administration (hydration volume, amount of sodium administered, amount of potassium administered, and magnesium supplementation), the laboratory data associated with diagnosis of SIADH (plasma concentration of ADH, plasma osmolality, urinary osmolality, urine sodium, serum creatinine, serum concentration of cortisol), presence of diabetes, and use of non-steroidal anti-inflammatory drugs, opioids, diuretics, proton pump inhibitors and antidepressants. The diagnostic criteria of SIADH was defined based on the Japanese guide for the diagnosis of SIADH as the following: hyponatremia $(<135 \mathrm{mEq} / \mathrm{L})$, increase in plasma concentration of antidiuretic hormone (ADH) (above limit of quantitation), low plasma osmolality $(<280 \mathrm{mOsm} / \mathrm{kg})$, concentrated urine $(>300$ $\mathrm{mOsm} / \mathrm{kg})$, natriuresis $(\geq 20 \mathrm{mEq} / \mathrm{L})$, normal renal function (serum creatinine lower than $1.2 \mathrm{mg} / \mathrm{dL}$ ), and normal adrenal function (serum concentration of cortisol higher than $6 \mu \mathrm{g} / \mathrm{dL}$ ), in addition to the absence of dehydration. ${ }^{11)}$

Statistical Analysis Categorical variables were analyzed using the chi-square test or Fisher's exact test. Ordinal variables were evaluated using the Cochran-Armitage test. Odds ratios and 95\% confidence intervals (CIs) of risk factors for grade 3 or 4 hyponatremia were calculated by using logistic regression models. Baseline variables with $p$-values $<0.05$ in univariate analyses were included in the multivariable logistic regression model. All $p$-values were determined using twosided tests, and differences with $p$-values $<0.05$ were considered statistically significant. All statistical analyses were performed using EZR (Saitama Medical Center, Jichi Medical University, Saitama, Japan), ${ }^{12)}$ which is a graphical user interface for R software (The R Foundation for Statistical Computing, Vienna, Austria) that serves as a modified version of $\mathrm{R}$ commander and includes common biostatistical functions.

\section{RESULTS}

Patient Characteristics A total of 733 patients were prescribed CDDP between the study period, but only 684 patients were assessed for eligibility. Among those, 178 patients met the exclusion criteria, 19 patients did not have measurements of their serum sodium concentrations, and 15 patients were lost to follow up. As a consequence, only 472 patients were included in the analysis (Fig. 1).

The characteristics of the patients are summarized in Table

Table 1. Patients' Demographics and Clinical Information

\begin{tabular}{|c|c|c|}
\hline$N$ & & 472 \\
\hline Age & & $62.5 \pm 10.7$ \\
\hline \multirow[t]{2}{*}{ Sex } & Male & $314(66.5)$ \\
\hline & Female & $158(33.5)$ \\
\hline \multirow[t]{11}{*}{ Tumor type } & Non small cell lung & $78(16.5)$ \\
\hline & Head and neck & $78(16.5)$ \\
\hline & Esophageal & $72(15.3)$ \\
\hline & Gastric & $61(12.9)$ \\
\hline & Cervical & $43(9.1)$ \\
\hline & Bladder & $40(8.5)$ \\
\hline & Small cell lung & $22(4.7)$ \\
\hline & Lymphoma & $16(3.4)$ \\
\hline & Endometrial & $15(3.2)$ \\
\hline & Neuroendocrine & $13(2.8)$ \\
\hline & Others & $34(7.2)$ \\
\hline Cisplatin dose $\left(\mathrm{mg} / \mathrm{m}^{2}\right)$ & & $67.7 \pm 13.9$ \\
\hline \multirow[t]{4}{*}{ Number of concomitant anticancer drug } & 0 & $35(7.4)$ \\
\hline & 1 & $363(76.9)$ \\
\hline & 2 & $56(11.9)$ \\
\hline & 3 & $18(3.8)$ \\
\hline Creatinine clearance $(\mathrm{mL} / \mathrm{min})$ & & $93.1 \pm 32.4$ \\
\hline Serum sodium concentration $(\mathrm{mEq} / \mathrm{L})$ & & $140.1 \pm 3.2$ \\
\hline Number of sodium measurements & & $4.3 \pm 2.7$ \\
\hline
\end{tabular}

Data are shown average \pm standard deviation or $n(\%)$.

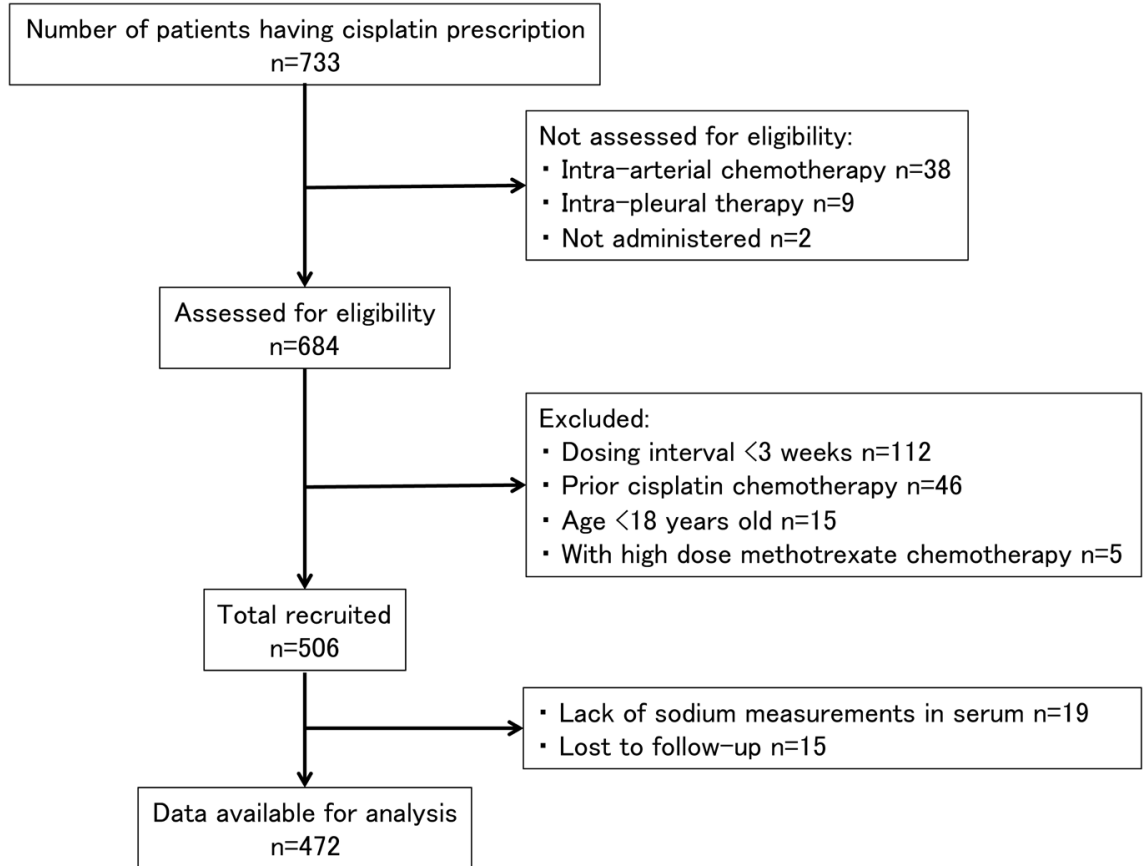

Fig. 1. Flow Chart of Patient Recruitment 
Table 2. Hydration Regimen on the Day of CDDP Administration

\begin{tabular}{|c|c|c|c|c|c|}
\hline & $N$ & Hydration volume (mL) & $\mathrm{Na}$ Administered (mEq) & K Administered (mEq) & Mg Supplementation \\
\hline Non small cell lung & 78 & $3324 \pm 559$ & $301 \pm 70$ & $20.2 \pm 17.3$ & $51(65.4)$ \\
\hline Head and neck & 78 & $3699 \pm 311$ & $294 \pm 65$ & $34.6 \pm 18.4$ & $73(93.6)$ \\
\hline Esophageal & 72 & $3148 \pm 486$ & $369 \pm 48$ & $15.0 \pm 11.4$ & $12(16.7)$ \\
\hline Gastric & 61 & $2844 \pm 547$ & $335 \pm 58$ & $18.9 \pm 15.6$ & $20(32.8)$ \\
\hline Cervical & 43 & $4395 \pm 306$ & $500 \pm 33$ & $29.3 \pm 3.6$ & $0(0.0)$ \\
\hline Bladder & 40 & $3117 \pm 526$ & $292 \pm 35$ & $20.3 \pm 4.7$ & $0(0.0)$ \\
\hline Small cell lung & 22 & $3083 \pm 467$ & $198 \pm 36$ & $31.4 \pm 4.7$ & $17(77.3)$ \\
\hline Lymphoma & 16 & $2704 \pm 890$ & $305 \pm 131$ & $4.7 \pm 10.6$ & $14(87.5)$ \\
\hline Endometrial & 15 & $3158 \pm 301$ & $188 \pm 81$ & $44.0 \pm 17.4$ & $1(6.7)$ \\
\hline Neuroendocrine & 13 & $3709 \pm 1102$ & $342 \pm 118$ & $27.2 \pm 13.2$ & $9(69.2)$ \\
\hline Others & 34 & $3370 \pm 705$ & $325 \pm 99$ & $27.7 \pm 12.5$ & $8(23.5)$ \\
\hline Total & 472 & $3353 \pm 679$ & $326 \pm 96$ & $23.9 \pm 16.0$ & $205(43.4)$ \\
\hline
\end{tabular}

Data are shown as average \pm standard deviation or $n(\%)$.

Table 3. Incidence of Hyponatremia in Patients with Different Cancers

\begin{tabular}{|c|c|c|c|c|c|c|}
\hline & \multirow{2}{*}{$N$} & \multirow{2}{*}{ All grades } & \multirow{2}{*}{ Grade 1} & \multirow{2}{*}{ Grade 3} & \multirow{2}{*}{ Grade 4} & $p$-Value \\
\hline & & & & & & Grade $3 / 4$ \\
\hline Non small cell lung & 78 & $58(74.4)$ & $51(65.4)$ & $7(9.0)$ & $0(0.0)$ & $0.759^{a)}$ \\
\hline Head and neck & 78 & $63(80.8)$ & $54(69.2)$ & $8(10.3)$ & $1(1.3)$ & $0.924^{a)}$ \\
\hline Esophageal & 72 & $52(72.2)$ & $39(54.2)$ & $10(13.9)$ & $3(4.2)$ & $0.043^{a)}$ \\
\hline Gastric & 61 & $29(47.5)$ & $26(42.6)$ & $2(3.3)$ & $1(1.6)$ & $0.187^{a)}$ \\
\hline Cervical & 43 & $11(25.6)$ & 9 (20.9) & $2(4.7)$ & $0(0.0)$ & $0.295^{b)}$ \\
\hline Bladder & 40 & $32(80.0)$ & $28(70.0)$ & $4(10.0)$ & $0(0.0)$ & $1^{b)}$ \\
\hline Small cell lung & 22 & $22(100.0)$ & $16(72.7)$ & $3(13.6)$ & $3(13.6)$ & $0.021^{b)}$ \\
\hline Lymphoma & 16 & $13(81.3)$ & $12(75.0)$ & $1(6.3)$ & $0(0.0)$ & $1^{b)}$ \\
\hline Endometrial & 15 & $2(13.3)$ & $1(6.7)$ & $1(6.7)$ & $0(0.0)$ & $1^{b)}$ \\
\hline Neuroendocrine & 13 & $13(100.0)$ & $12(92.3)$ & $1(7.7)$ & $0(0.0)$ & $1^{b)}$ \\
\hline Others & 34 & $22(64.7)$ & $19(55.9)$ & $3(8.8)$ & $0(0.0)$ & $1^{b)}$ \\
\hline Total & 472 & $317(67.2)$ & $267(56.6)$ & $42(8.9)$ & $8(1.7)$ & \\
\hline
\end{tabular}

Data are shown $n(\%)$. $p$-Values are for the comparison of grades 3 and 4 hyponatremia incidence between each cancer group and all others. $a$ ) chi-square test, $b$ ) Fisher's exact test.

1. Mean age ( \pm standard deviation) was $62.5( \pm 10.7)$ years, and $66.5 \%$ of the patients were men. Several tumor types were observed, such as non-small cell lung $(16.5 \%)$, head and neck $(16.5 \%)$, esophageal $(15.3 \%)$, gastric $(12.9 \%)$, cervical $(9.1 \%)$, bladder (8.5\%), small cell lung (4.7\%), lymphoma (3.4\%), endometrial $(3.2 \%)$, and neuroendocrine $(2.8 \%)$ tumors. The mean CDDP dose ( \pm standard deviation) was $67.7( \pm 13.9)$ $\mathrm{mg} / \mathrm{m}^{2}$. The baseline mean of serum sodium concentration ( \pm standard deviation) was $140.1( \pm 3.2) \mathrm{mEq} / \mathrm{L}$, and the mean of the number of sodium measurements in the follow up period ( \pm standard deviation) was $4.3( \pm 2.7)$ times. Hydration regimen on the day of CDDP administration is described in Table 2. The mean values ( \pm standard deviation) of hydration volume, amount of sodium administered, and amount of potassium administered among all subjects were $3353 \pm 679 \mathrm{~mL}$, $326 \pm 96$, and $23.9 \pm 16.0 \mathrm{mEq}$, respectively. The proportion of patients who were supplied magnesium was $43.4 \%$.

Incidences of Hyponatremia The incidence of hyponatremia following CDDP administration was $67.2 \%$, and specifically for grade 4 and 3 were 1.7 and $8.9 \%$, respectively. Grade 1 hyponatremia was observed in $56.6 \%$ of the study subjects. The median for day of grade $\geq 3$ hyponatremia incidence was 7 (interquartile range: $5-8$ ). Four patients $(0.9 \%)$ stopped CDDP chemotherapy because of severe hyponatremia.
Frequencies of hyponatremia above grade 3 in patients with small cell lung cancer (SCLC) $(27.3 \%)$ and esophageal cancer (18.1\%) were significantly higher than those of patients with other types of cancer ( $p=0.021$ and 0.043 , respectively; Table 3). In the experimental group, proportions of $\mathrm{ADH}$ plasma concentration, plasma osmolality, urinary osmolality, urine sodium, serum creatinine, and serum cortisol concentration were 4/50 (8\%), $8 / 50(16 \%), 10 / 50(20 \%), 17 / 50(34 \%), 50 / 50$ $(100 \%)$, and $3 / 50(6 \%)$, respectively. The proportions of each item meeting the criteria of SIADH were $4 / 4,8 / 8,7 / 10,16 / 17$, $39 / 50$, and $3 / 3$, respectively. Only three patients were measured for all clinical parameters associated with SIADH. Two patients, one with SCLC and the other with neuroendocrine cancer, met all laboratory data criteria. The other patient had esophageal cancer and failed to meet the criteria because of the development renal failure. In addition, a patient with SCLC did not have cortisol measurements, but all other items met the criteria.

Univariate Analysis The results of univariate logistic regression analysis are shown in Table 4. Age ( $\geq 65$ years), SCLC, esophageal cancer, and lower baseline of serum sodium concentration $(<138 \mathrm{mEq} / \mathrm{L})$ were identified as risk factors for grade $\geq 3$ hyponatremia. Serum albumin before treatment was measured in $97.7 \%$ of study subjects. Hypoalbuminemia 
Table 4. Univariate Logistic Regression Analysis for Grades 3 and 4 Hyponatremia

\begin{tabular}{|c|c|c|c|c|c|c|}
\hline & & Grade $0 / 1(n=422)$ & Grade $3 / 4(n=50)$ & Crude OR & $95 \% \mathrm{CI}$ & $p$-Value \\
\hline \multirow[t]{2}{*}{ Age } & $\geq 65$ & 194 & 32 & 2.09 & $1.14-3.84$ & $<0.05$ \\
\hline & $<65$ & 228 & 18 & 1 (ref.) & & \\
\hline \multirow[t]{2}{*}{ Sex } & male & 275 & 39 & 1.90 & $0.94-3.81$ & 0.07 \\
\hline & female & 147 & 11 & 1 (ref.) & & \\
\hline \multirow[t]{3}{*}{ Tumor type } & SCLC & 16 & 6 & 4.20 & $1.53-11.50$ & $<0.005$ \\
\hline & Esophageal & 59 & 13 & 2.47 & $1.22-4.99$ & $<0.05$ \\
\hline & Others & 347 & 31 & 1 (ref.) & & \\
\hline \multirow[t]{2}{*}{ Cisplatin dose $\left(\mathrm{mg} / \mathrm{m}^{2}\right)$} & $\geq 70$ & 186 & 29 & 1.75 & $0.97-3.17$ & 0.06 \\
\hline & $<70$ & 236 & 21 & 1 (ref.) & & \\
\hline \multirow[t]{2}{*}{ Hydration volume (mL) } & $\geq 3353$ & 219 & 24 & 1 (ref.) & & \\
\hline & $<3353$ & 203 & 26 & 1.17 & $0.65-2.10$ & 0.60 \\
\hline \multirow[t]{2}{*}{$\mathrm{Na}$ administered (mEq) } & $\geq 326$ & 241 & 27 & 1 (ref.) & & \\
\hline & $<326$ & 181 & 23 & 1.13 & $0.63-2.04$ & 0.68 \\
\hline \multirow[t]{2}{*}{$\mathrm{K}$ administered (mEq) } & $\geq 23.9$ & 223 & 29 & 1 (ref.) & & \\
\hline & $<23.9$ & 199 & 21 & 0.81 & $0.45-1.47$ & 0.49 \\
\hline Mg supplementation & & 182 & 23 & 1.12 & $0.62-2.02$ & 0.70 \\
\hline \multirow[t]{4}{*}{ Number of concomitant anticancer drug } & 3 & 18 & 0 & - & - & - \\
\hline & 2 & 49 & 7 & 1.52 & $0.367-6.330$ & 0.56 \\
\hline & 1 & 323 & 40 & 1.32 & $0.387-4.510$ & 0.66 \\
\hline & 0 & 32 & 3 & 1 (ref.) & & \\
\hline \multirow[t]{6}{*}{ Concomitant drug } & NSAID & 77 & 8 & 0.85 & $0.39-1.89$ & 0.70 \\
\hline & Opioid & 51 & 9 & 1.60 & $0.73-3.48$ & 0.24 \\
\hline & Diuretic & 27 & 6 & 1.99 & $0.78-5.10$ & 0.15 \\
\hline & Vinca alkaloid & 3 & 67 & 0.34 & $0.10-1.12$ & 0.08 \\
\hline & PPI & 140 & 15 & 0.86 & $0.46-1.63$ & 0.65 \\
\hline & Antidepressant & 10 & 0 & - & - & - \\
\hline \multirow[t]{2}{*}{ Creatinine clearance (mL/min) } & $\geq 60$ & 375 & 41 & 1 (ref.) & & \\
\hline & $<60$ & 47 & 9 & 1.75 & $0.80-3.83$ & 0.16 \\
\hline Diabetes mellitus & & 61 & 11 & 1.66 & $0.80-3.41$ & 0.17 \\
\hline \multirow[t]{2}{*}{ Serum sodium } & $\geq 138$ & 377 & 27 & 1 (ref.) & & \\
\hline & $<138$ & 45 & 23 & 7.14 & $3.78-13.50$ & $<0.001$ \\
\hline
\end{tabular}

OR, Odds ratio; CI, Confidence interval; SCLC, Small cell lung cancer; NSAID, Non-steroidal anti-inflammatory drug; PPI, Proton pump inhibitor.

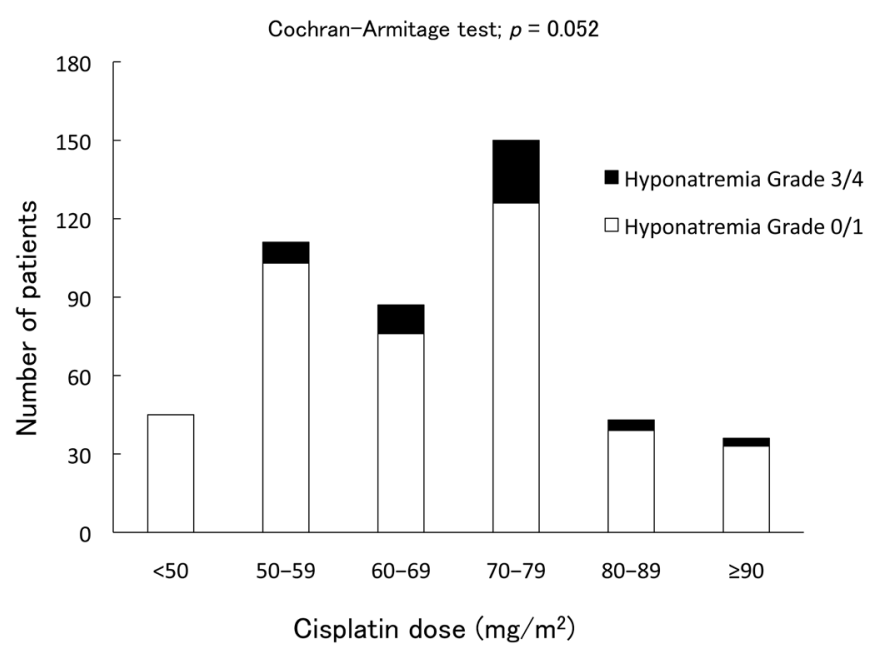

Fig. 2. The Association between Cisplatin Dose and Incidence of Hyponatremia

$(<3.0 \mathrm{mg} / \mathrm{dL})$ was observed in 7/49 of experimental group and $32 / 412$ of control group. Hypoalbuminemia revealed no association with development of grade $\geq 3$ hyponatremia (crude odds ratio $1.98,95 \%$ CI $0.82-4.76, p=0.13$ ).

Association between CDDP Dose and Grade 3 or 4
Hyponatremia There was no significant difference in the development of grade $\geq 3$ hyponatremia between high-dose $\left(\geq 70 \mathrm{mg} / \mathrm{m}^{2}\right)$ and low-dose $\left(<70 \mathrm{mg} / \mathrm{m}^{2}\right)$ CDDP. We conducted an additional analysis to examine the dose-dependent increase in the development of grade $\geq 3$ hyponatremia. The proportions of hyponatremia above grade 3 were $0 / 45(0.0 \%), 8 / 111$ (7.2\%), 11/87 (12.6\%), 24/150 (16.0\%), 4/43 (9.3\%), and 3/36 (8.3\%) for CDDP doses of $<50,50-59,60-69,70-79,80-89$, and $\geq 90 \mathrm{mg} / \mathrm{m}^{2}$, respectively (Fig. 2). There was no CDDP dose-dependent increase in the development of grade $\geq 3$ hyponatremia $(p=0.052)$.

Multivariate Logistic Regression Analysis A multivariate logistic regression analysis assessed the contribution of each individual risk factor to the onset of grade 3 or 4 hyponatremia. Both SCLC (adjusted odds ratio, 3.26; 95\%CI, 1.07-10.00), and lower baseline of serum sodium concentration $(<138 \mathrm{mEq} / \mathrm{L})$ (adjusted odds ratio, 6.18; 95\%CI, 3.21-11.90) were significantly associated with an increased risk for severe hyponatremia (Fig. 3).

Subanalysis of Patients with SCLC The subanalysis assessed the contribution of concomitant anticancer drugs and adverse events related to chemotherapy in the presence of grade $\geq 3$ hyponatremia in patients with SCLC. Nineteen patients received etoposide (VP-16) with CDDP, and 3 patients were administered with irinotecan (CPT-11). The incidence of 


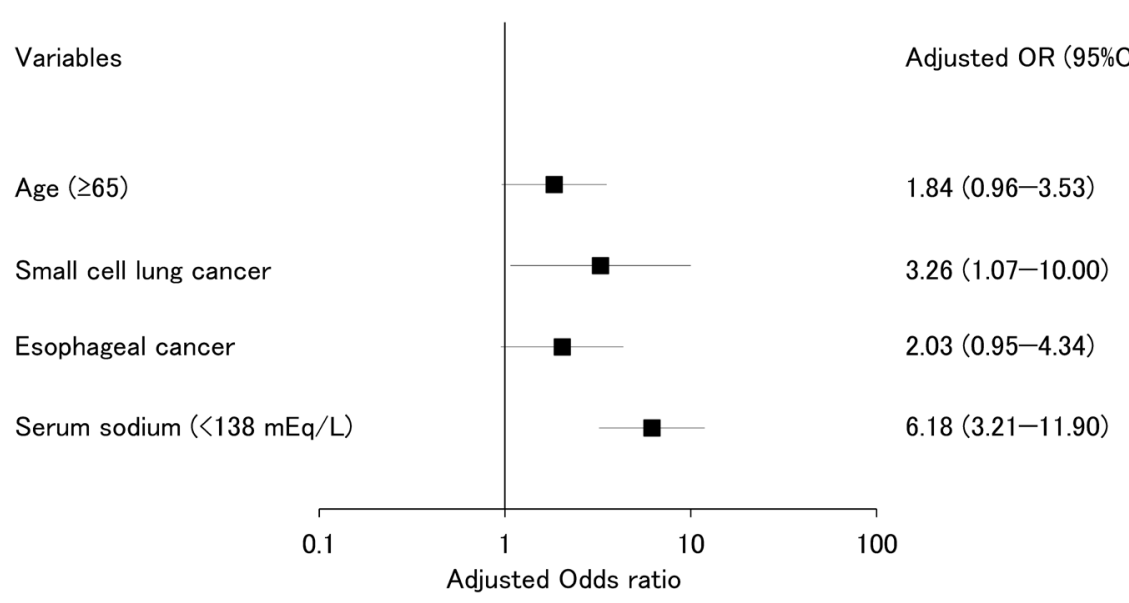

Fig. 3. Multi-Variable Logistic Regression Analysis for Severe Hyponatremia

Table 5. Chemotherapy Related Adverse Events on Case Group

\begin{tabular}{lcccc}
\hline \hline & All patients & \multicolumn{3}{c}{ Tumor type } \\
\cline { 3 - 5 } & & SCLC & Other & $p$-Value \\
\hline$N$ & 50 & 6 & 44 & $16(36.4)$ \\
Nausea $(\geq$ grade 2) & $18(36.0)$ & $2(33.3)$ & $24(54.6)$ & 1 \\
Anorexia $(\geq$ grade 2) & $27(54.0)$ & $0(0.0)$ & $1(2.3)$ & 1 \\
Vomiting $(\geq$ grade 2) & $1(2.0)$ & $0(0.0)$ & $7(15.9)$ & 0.576 \\
Diarrhea $(\geq$ grade 2) & $7(14.0)$ & $0(0.0)$ & $9(20.5)$ & 0.576 \\
Renal dysfunction $(\geq$ grade 2) & $9(18.0)$ & & \\
\hline
\end{tabular}

Data are shown $n(\%)$, SCLC, Small cell lung cancer. Groups were compared using Fisher's exact test.

grade $\geq 3$ hyponatremia was 31.6 and $0 \%$ in patients receiving VP-16 and CPT-11, respectively $(p=0.532)$. VP-16 was also administered to 11 patients with neuroendocrine cancer and 4 patients with other cancer types. Of the patients who received VP-16, 6.7\% developed grade $\geq 3$ hyponatremia. Although not significant, patients who received VP-16 with SCLC developed grade $\geq 3$ hyponatremia more frequently than patients with other cancer types receiving VP-16 $(p=0.104)$.

Among patients with grade $\geq 3$ hyponatremia, the incidence of adverse events that possibly caused hyponatremia were compared between patients with SCLC and with other cancers. Although not significant, patients with SCLC were less likely to develop grade $\geq 2$ diarrhea, or renal failure, compared to patients with other cancers $(0.0$ vs. 15.9 and 0.0 vs. $20.5 \%$, respectively). There was no significant difference in the incidences of grade 2 or higher nausea, anorexia, and vomiting (Table 5).

\section{DISCUSSION}

This study is the first one to investigate independent risk factors for severe hyponatremia, following first CDDP administration in patients suffering from various types of cancer. Thus, frequencies and independent risk factors of hyponatremia on each cancer type were investigated. As a result, SCLC and lower serum sodium concentrations $(<138 \mathrm{mEq} / \mathrm{L})$ were identified as independent risk factors for hyponatremia above grade 3 .

The incidence of severe hyponatremia following CDDP administration was highly frequent and differed by cut-off level of serum sodium or study population. Some retrospective observational studies reported that frequency of severe hyponatremia was $15-41 \%{ }^{13-15)}$; meanwhile, a recent integrated analysis of clinical trials stated a frequency of $13.5 \%{ }^{10)}$ Some case reports showed that severe hyponatremia following CDDP administration induced cessation of chemotherapy. ${ }^{16,17)}$ Ezoe et al. evidenced that the incidence of severe hyponatremia following platinum-based chemotherapy varied among different types of cancer (5.4-19.9\%), and grade 4 hyponatremia was significantly higher in patients with SCLC than others. ${ }^{10)}$ Relative to previous investigations, the present study showed a comparable incidence of grade $\geq 3$ hyponatremia.

A study reported that a higher dose of CDDP, number of concomitant anticancer drugs, SCLC, and combination with amrubicin or irinotecan were associated with the risk of severe hyponatremia. ${ }^{10)}$ In contrast, an investigation on head and neck cancers showed that patients who received mannitol had higher incidence of grade 3 hyponatremia than patients who did not, but the independent risk factors were unclear because no multivariate analysis was conducted. ${ }^{15)}$ Ochi et al. analyzed the risk factors of severe hyponatremia following CDDP administration on lung cancer patients by a multivariable logistic regression model. ${ }^{14)}$ They observed that the use of generic CDDP increased the incidence of hyponatremia, compared to the brand-name drug, and concluded that the drug formulation constituted an independent risk factor for the condition. However, they did not consider the histological type of lung cancer. Our results indicated that independent risk factors of hyponatremia above grade 3 following first CDDP administration were SCLC and lower sodium concentrations in the 
serum $(<138 \mathrm{mEq} / \mathrm{L})$.

Previous studies reported that the main etiology of hyponatremia following CDDP administration was RSW associated with renal failure or SIADH. ${ }^{8,9,17)}$ The mechanism of SIADH caused by CDDP has not been clarified, but it is thought that CDDP stimulates ADH secretion. ${ }^{18)}$ The mechanism of RSW caused by CDDP is thought to be due to CDDP directly damaging renal tubules and interfering with sodium reabsorption. In this study, SIADH was suspected in two SCLC patients and a neuroendocrine cancer patient with grade $\geq 3$ hyponatremia based on laboratory data associated with SIADH. Although the other SCLC patients missed too many clinical examinations to diagnose SIADH, there were no renal failure occurrences or adverse events related to dehydration, such as vomiting or diarrhea. Because of this, it is possible that SIADH could have been implicated in these patients. It is known that SIADH is occasionally caused by ectopic production of ADH by the tumor. Cancers including adenocarcinomas and squamous cell carcinomas are known to produce and release ADH, especially in SCLC. ${ }^{19)}$ The frequency of SIADH in SCLC patients was reported to be about $15 \% .^{18,19)}$ Moreover, in this study, the frequency of hyponatremia in SCLC patients was high. Some studies which examined the association between prognosis and hyponatremia on SCLC patients, revealed that a frequency of $17.9-43.7 \%$ study patients had hyponatremia. ${ }^{20,21)}$ Another report which examined patients with various types of cancer observed that the proportion of hyponatremia in lung cancer patients was the highest $(40.2 \%)$, followed by prostate, pancreatic, and hepatic cancers. $\left.{ }^{4}\right)$ Therefore, the reason for the high incidence of severe hyponatremia in SCLC patients was associated with the cancer type, in addition to the effect of CDDP. However, it was difficult to assess extracellular fluid volume and laboratory data for diagnosis of SIADH in this retrospective study. Further studies with appropriate assessment of hyponatremia etiology are warranted.

In patients with SCLC, hyponatremia above grade 3 was observed only in subjects receiving VP-16. However, the effect of concomitant anticancer agents could not be adequately examined because the number of patients receiving CPT-11 was small. The difference in severe hyponatremia incidence after receiving VP-16 between patients suffering from SCLC and other cancer types was not significant, although higher numbers were noted in the first group. Therefore, our results suggested that the cancer type may have an impact. Furthermore, the influence of concomitant anticancer drugs should be investigated in the future.

It was clear that low serum sodium concentration $(<138 \mathrm{mEq} / \mathrm{L})$ before treatment was identified as an independent risk factor because a decrease in serum sodium concentration was observed after CDDP administration. Ochi et al. showed that the median value of serum sodium concentration reduction was $6.0 \mathrm{mEq} / \mathrm{L} .{ }^{14)}$ Boku et al. showed a reduction of $5-15 \mathrm{mEq} / \mathrm{L}$ in most of the patients after daily CDDP administration for $5 \mathrm{~d}^{13)}$ There was a possibility that a temporary increase in circulating plasma volume by hydration with CDDP administration was associated with a decrease in serum sodium concentration. In this study, hydration regimen was different among types of cancer, and the amount of sodium administered to SCLC patients was lower than the amount administered to patients with other cancer types. However, there was no statistically significant association between grade $\geq 3$ hyponatremia and hydration volume, amount of sodium administered, amount of potassium administered, or magnesium supplementation. A previous study also showed that the amount of sodium administered was not associated with onset of severe hyponatremia. ${ }^{13)}$

The association between development of hyponatremia above grade 3 and CDDP dose has been unclear. One recent study indicated that patients receiving a high dose of CDDP $\left(\geq 20 \mathrm{mg} / \mathrm{m}^{2} /\right.$ week) had a higher proportion of grade 4 hyponatremia than those receiving a lower dose $\left(<20 \mathrm{mg} / \mathrm{m}^{2} /\right.$ week $)$ using univariate analysis. ${ }^{10)}$ On the contrary, another study showed that there was no association between CDDP dose and development of grade $\geq 3$ hyponatremia or the lowest serum sodium value. ${ }^{14)}$ In our study, dose-dependent increase in development of grade $\geq 3$ hyponatremia was not observed, but no grade $\geq 3$ hyponatremia developed in the lowest CDDP dose group $\left(<50 \mathrm{mg} / \mathrm{m}^{2}\right)$. Although it was suggested that low dose of CDDP might be unlikely to cause severe hyponatremia, the effect was not clear because of the small sample size of the lowest dose group. In addition, our study could not explore the effect of cumulative dose and number of doses because study population was only examined at the first cycle of CDDP treatment. Additional studies are needed to clarify the effect of CDDP dose to hyponatremia.

This retrospective study had several limitations. First, the dates of measurement and frequency of serum sodium concentrations differed among patients. Second, the influence of concomitant anticancer agents could not be sufficiently examined. Third, etiologies of hyponatremia could not be clarified because measurements of the laboratory data associated with diagnosis of SIADH were only performed in some patients, and dietary intake was not adequately examined. These limitations indicated that a prospective study is needed to confirm our result, and to clarify the influence of concomitant anticancer drugs, dietary intake, and SIADH.

Therefore, the present study revealed that SCLC and lower serum sodium concentrations $(<138 \mathrm{mEq} / \mathrm{L})$ were independent risk factors for severe hyponatremia following first CDDP administration. Hyponatremia was frequently observed under these conditions, and in cases of severe hyponatremia, some patients were required to discontinue CDDP chemotherapy. Therefore, early detection and treatment of hyponatremia are needed to continue CDDP chemotherapy. In particular, more oversight of serum sodium concentrations after CDDP chemotherapy is necessary in patients with SCLC who have lower serum sodium concentrations.

Conflict of Interest The authors declare no conflict of interest.

\section{REFERENCES}

1) Berardi R, Rinaldi S, Caramanti M, Grohè C, Santoni M, Morgese F, Torniai M, Savini A, Fiordoliva I, Cascinu S. Hyponatremia in cancer patients: Time for a new approach. Crit. Rev. Oncol. Hematol., 102, 15-25 (2016).

2) Doshi SM, Shah P, Lei X, Lahoti A, Salahudeen AK. Hyponatremia in hospitalized cancer patients and its impact on clinical outcomes. Am. J. Kidney Dis., 59, 222-228 (2012)

3) Berghmans T, Paesmans M, Body JJ. A prospective study on hyponatraemia in medical cancer patients: epidemiology, aetiology and 
differential diagnosis. Support. Care Cancer, 8, 192-197 (2000).

4) Abu Zeinah GF, Al-Kindi SG, Hassan AA, Allam A. Hyponatraemia in cancer: association with type of cancer and mortality. Eur. J. Cancer Care (Engl.), 24, 224-231 (2015).

5) Dasari S, Tchounwou PB. Cisplatin in cancer therapy: molecular mechanisms of action. Eur. J. Pharmacol., 740, 364-378 (2014).

6) Florea AM, Büsselberg D. Cisplatin as an anti-tumor drug: cellular mechanisms of activity, drug resistance and induced side effects. Cancers (Basel), 3, 1351-1371 (2011).

7) Lee YK, Shin DM. Renal salt wasting in patients treated with highdose cisplatin, etoposide, and mitomycin in patients with advanced non-small cell lung cancer. Korean J. Intern. Med., 7, 118-121 (1992).

8) $\overline{\text { Iyer } \mathrm{AV}}$, Krasnow SH, Dufour DR, Arcenas AS. Sodium-wasting nephropathy caused by cisplatin in a patient with small-cell lung cancer. Clin. Lung Cancer, 5, 187-189 (2003).

9) Daugaard G, Abildgaard U, Holstein-Rathlou NH, Bruunshuus I, Bucher D, Leyssac PP. Renal tubular function in patients treated with high-dose cisplatin. Clin. Pharmacol. Ther., 44, 164-172 (1988).

10) Ezoe Y, Mizusawa J, Katayama H, Kataoka K, Muto M. An integrated analysis of hyponatremia in cancer patients receiving platinum-based or nonplatinum-based chemotherapy in clinical trials (JCOG1405-A). Oncotarget, 9, 6595-6606 (2017)

11) The Japanese Society for Hypothalamic and Pituitary tumors. "Guide for the diagnosis and treatment of SIADH.": 〈http://square. umin.ac.jp/kasuitai/guidance/SIADH.pdf), accessed 20 July, 2019.

12) Kanda Y. Investigation of the freely available easy-to-use software 'EZR' for medical statistics. Bone Marrow Transplant., 48, 452-458 (2013).

13) Boku N, Ohtsu A, Nagashima F, Muto M, Shinkai T, Yoshida S. Retrospective study of hyponatremia in gastric cancer patients treated with a combination chemotherapy of 5-fluorouracil and cis- platin: a possible warning sign of severe hematological toxicities? Jpn. J. Clin. Oncol., 31, 382-387 (2001).

14) Ochi N, Yamane $\mathrm{H}$, Hotta $\mathrm{K}$, Fujii $\mathrm{H}$, Isozaki H, Honda $\mathrm{Y}$, Yamagishi T, Kubo T, Tanimoto M, Kiura K, Takigawa N. Cisplatin-induced hyponatremia in malignancy: comparison between brand-name and generic formulation. Drug. Des. Devel. Ther, 8, 2401-2408 (2014).

15) McKibbin T, Cheng LL, Kim S, Steuer CE, Owonikoko TK, Khuri FR, Shin DM, Saba NF. Mannitol to prevent cisplatin-induced nephrotoxicity in patients with squamous cell cancer of the head and neck (SCCHN) receiving concurrent therapy. Support. Care Cancer, 24, 1789-1793 (2016).

16) El Weshi A, Thieblemont C, Cottin V, Barbet N, Catimel G. Cisplatin-induced hyponatremia and renal sodium wasting. Acta Oncol., 34, 264-265 (1995)

17) Kwon DY, Han GH, Ulak R, Ki KD, Lee JM, Lee SK. Syndrome of inappropriate antidiuretic hormone secretion following irinotecancisplatin administration as a treatment for recurrent ovarian clear cell carcinoma. Obstet. Gynecol. Sci., 60, 115-117 (2017).

18) Castillo JJ, Vincent M, Justice E. Diagnosis and management of hyponatremia in cancer patients. Oncologist, 17, 756-765 (2012).

19) Sørensen JB, Andersen MK, Hansen HH. Syndrome of inappropriate secretion of antidiuretic hormone (SIADH) in malignant disease. J. Intern. Med., 238, 97-110 (1995).

20) Hansen O, Sørensen P, Hansen KH. The occurrence of hyponatremia in SCLC and the influence on prognosis: a retrospective study of 453 patients treated in a single institution in a 10 -year period. Lung Cancer, 68, 111-114 (2010).

21) Tiseo M, Buti S, Boni L, Mattioni R, Ardizzoni A. Prognostic role of hyponatremia in 564 small cell lung cancer patients treated with topotecan. Lung Cancer, 86, 91-95 (2014). 